\title{
Automatic Trajectory Planning for Deep Brain Stimulation: A Feasibility Study
}

\author{
Ellen J.L. Brunenberg ${ }^{1}$, Anna Vilanova ${ }^{1}$, Veerle Visser-Vandewalle ${ }^{2}$, \\ Yasin Temel ${ }^{2}$, Linda Ackermans ${ }^{2}$, Bram Platel ${ }^{3}$, \\ and Bart M. ter Haar Romeny ${ }^{1}$ \\ ${ }^{1}$ Department of Biomedical Engineering, \\ Eindhoven University of Technology, The Netherlands \\ e.j.l.brunenberg@tue.nl \\ ${ }^{2}$ Department of Neurosurgery \\ ${ }^{3}$ Department of Biomedical Engineering, \\ Maastricht University Hospital, The Netherlands
}

\begin{abstract}
DBS for Parkinson's disease involves an extensive planning to find a suitable electrode implantation path to the selected target. We have investigated the feasibility of improving the conventional planning with an automatic calculation of possible paths in $3 \mathrm{D}$. This requires the segmentation of anatomical structures. Subsequently, the paths are calculated and visualized. After selection of a suitable path, the settings for the stereotactic frame are determined. A qualitative evaluation has shown that automatic avoidance of critical structures is feasible. The participating neurosurgeons estimate the time gain to be around 30 minutes.
\end{abstract}

\section{Introduction}

Parkinson's disease patients suffer from motor symptoms, caused by the loss of neurons and the subsequent subthalamic nucleus (STN) burst activity. In the early stage of the disease, patients are treated with dopamine. However, longterm drug taking usually results in severe side-effects. As a solution, deep brain stimulation (DBS) has been introduced [1. This involves the implantation of electrodes, which, according to the general consensus, modulate STN activity.

Currently, the procedure can take more than eight hours. In our opinion, this could be shortened by refining the preoperative planning of the implantation trajectories. After indirect targeting of the STN, the conventional planning comprises manual selection of an entry point. The resulting path is examined slice by slice, to ensure that blood vessels, ventricles, and sulci are avoided. This is typically repeated a couple of times and can last more than one hour.

In previously developed planning systems for neurosurgery, often a great deal of user interaction is required. We would like to investigate the feasibility of automatically avoiding critical structures and shortening the path planning. After segmentation of the structures to be avoided, our contribution is the automatic calculation of possible paths to the STN in 3D. The resulting paths are then visualized, enabling the neurosurgeon to control their quality and select one. 
Section 2 discusses previous research. Section 3 presents an overview of the algorithm, after which Section 4 focuses on segmentation. In Section 5 , the path calculation is discussed, and the results are presented in Section 6. The validation can be found in Section 7 and finally some conclusions are given in Section 8 .

\section{Previous Work}

Many of the existing planning algorithms require manual selection of entry points, for example those of Nowinski et al. 22 and Lee et al. 3. Some only visualize the resulting path, others can also render target lesions and virtual tools. On the other hand, Bourbakis and Awad [4, Fujii et al. 5], and Vaillant et al. 6] describe elaborate path calculation algorithms. However, these require the manual assignment of tissue importance values, which is not very intuitive.

To our knowledge, there is no algorithm in literature that calculates safe paths automatically, without demanding manual setting of a complex cost function. We propose a possibility to select the costs easily by choosing two safety margins.

\section{Method Overview}

The proposal comprises anatomical segmentations, calculation of possible paths, and interactive visualization of the results. The desired pipeline is shown in Figure 1. The program separates MR and CT processing and is subdivided further to distinguish between user interaction and expensive calculations.

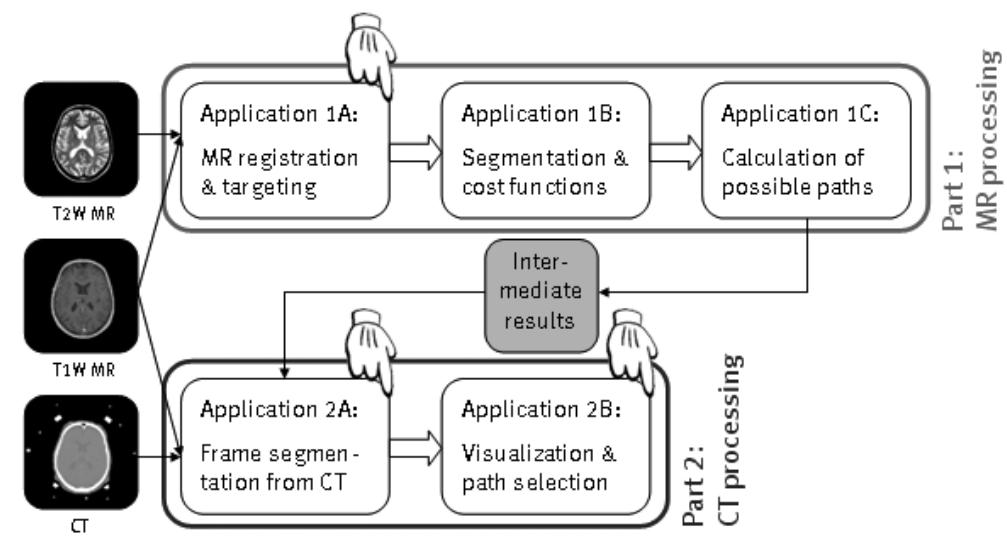

Fig. 1. Overview of the path planning algorithm with MR and CT processing parts. The hands indicate required interaction.

To find a safe path, the target and structures to be avoided, namely the blood vessels, ventricles, and sulci of the cortex, should be known. The corresponding calculations only need the MR images taken some days preoperatively. The 
developed MR processing part consists of three steps: registration and target selection, segmentation, and path calculation.

Contrast-enhanced T1- and T2-weighted MR and stereotactic CT have been collected for 4 patients. The MR images are registered manually and indirect atlas-based targeting of the STN is performed. Because these operations require user interaction, they are together in part $1 \mathrm{~A}$. In part $1 \mathrm{~B}$, the critical structures are segmented automatically. It is necessary to evade the blood vessels, also the small ones in the sulci of the cortex, in order to prevent hemorrhages. The ventricles should not be crossed to remain in control of the path, since the electrode is flexible. Subsequently, in part 1C, the target points and anatomical segmentations are used to calculate the possible paths for each side of the brain.

The CT processing is designed to be used on the operation day itself. It consists of two steps, both demanding interaction.

Part 2A involves manual registration of CT and MR. The frame is then segmented, and the parameters for the coordinate transformation from image space to frame space are calculated. The final part, $2 \mathrm{~B}$, visualizes the possible paths in $3 \mathrm{D}$, together with the segmented structures. The security margins for the vessels and the ventricles can be changed interactively. Eventually, the most suitable paths can be chosen and inspected by the surgeon in probe's eye view.

For avoiding critical structures the blocks 1B, 1C, and 2B are of interest.

\section{Segmentation of Anatomical Structures}

To segment the blood vessels, ventricles and gyri, we use several existing methods which, to our knowledge, have not been used in this context before. In advance, the MR images are preprocessed using coherence-enhancing diffusion [7], in order to decrease the image noise while preserving edges.

For ventricle segmentation, we combine a method by Géraud, Bloch and Maître 8] that involves thresholding and mathematical morphology with a method from Schnack et al. 9], using region-growing. An ROI which definitely contains (part of) the ventricles is automatically defined on the MR. We segment the CSF using thresholding and eliminate noise by erosion followed by dilation to reconstruct the ventricle structure but stay inside its boundaries. Finally, the remaining points are used as seeds for gray value-based region-growing. The segmented ventricles can be seen in Figure2 (left).

We have adapted Géraud's method [8] for brain tissue segmentation to our data. We use erosion and dilation, thresholding, connected components, and masking of T2- with results from T1-weighted MR. Afterwards, gyri and sulci are separated using the technique described by Lohmann 10. The brain is closed and a depth level intersection with the original segmentation is calculated. The result can be seen in Figure 2 (middle). The gyri are then divided into two hemispheres and restricted to the frontal lobe, to avoid entering the motor cortex.

For the enhancement of blood vessels we use the vesselness measure by Frangi et al. 11. After selecting the maximum response over 4 scales $(0.75-3 \mathrm{~mm})$, the image is thresholded to lose noise. To avoid false detection of vessels around 

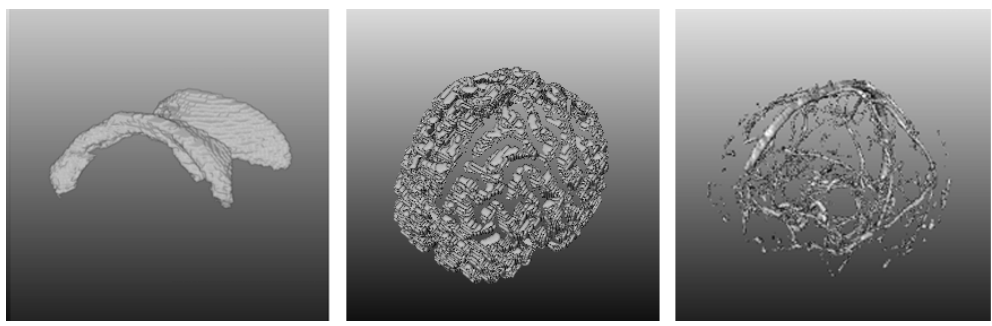

Fig. 2. Segmentations of anatomical structures. From left to right, the ventricles, gyri, and blood vessels can be seen.

the orbitae, the vessel image is masked with a slightly dilated segmentation of the brain tissue. The result can be seen in Figure 2 (right).

The segmentations are calculated fully automatically in MeVisLab. Due to the non-optimal implementation of the multi-scale vesselness, this takes around 60 minutes using a Pentium $43.06 \mathrm{GHz}$ processor and $1 \mathrm{~GB}$ of working memory.

\section{Path Planning}

The automatic calculation of safe entry points is the most distinguishing part of this work. A safe path has to avoid blood vessels, ventricles, and sulci, should begin in front of the motor cortex (to avoid epilepsy or motor deficits) but behind the hairline (for cosmetic reasons), and should be safe up to a small distance below the target, to enable deeper insertion.

To a certain extent, it is valid that the further the path stays from the vessels and ventricles, the safer it is. Therefore, a Euclidean distance transform was calculated to obtain two cost functions. These distance maps were thresholded on the size of the set of electrodes that are implanted, in our case $3 \mathrm{~mm}$, in order to have a value of 0 inside the segmented structure and the extra margin.

Existing studies on automatic planning applications require a lot of interaction for complex cost functions. We encountered basically two directions, one based on Dijkstra's path searching principle 12 and one on wave front propagation. However, these are not completely suitable for our application. First of all, they only find the minimum cost path, while we decided to determine every possibility and leave the choice to the surgeon. Furthermore, they need one proper cost function. We would have to add up the costs for the vessels and those for the ventricles in some artificial way, using extra normalization parameters. A linear cost accumulation also leads to the fact that almost hitting a vessel once gives the same cost as crossing the same vessel more often at a safer distance, which is not desirable. Finally, the paths proposed by the traditional methods are not restricted to straight lines, though necessary for our application. Therefore, we propose a very intuitive and easy-to-interact-with method.

Only pixels belonging to the frontal lobe gyri segmentation are considered to be possible entry points. For each of those, a straight line to the target position 
and $5 \mathrm{~mm}$ beyond is traced. Each point along this line is checked, calculating its distance to the vessels and ventricles using trilinear interpolation. If something is hit, e.g. if one of the distance maps is 0 at this point, the path is unsafe and therefore rejected. For all remaining paths, the minimum distance to a vessel and the minimum distance to a ventricle are stored. This enables the neurosurgeon to limit his options to safer paths.

Searching in a random list of entry points is of the order $\mathcal{O}(l)$, where $l$ is the total number of possible paths and will probably not be interactive. A possible solution lies in using a $2 \mathrm{D}$ ordered array. Each entry point is then stored in a certain bin according to its minimum distance to a vessel and to the ventricles. For example, the entry point for a path with a minimum distance of $1.4 \mathrm{~mm}$ to a vessel and $3.2 \mathrm{~mm}$ to the ventricles is classified in the bin belonging to a vessel distance interval of $[1.00,1.50) \mathrm{mm}$ and a ventricle distance of $[3.00,3.50) \mathrm{mm}$.

In this way, paths with distances higher than chosen thresholds can be proposed without further examination, while only the paths in bins at the margins have to be considered in detail. This is faster than a normal list search, accounting for an order of $\mathcal{O}(1+k)$, where $k$ is the number of paths in a bin, which should be much smaller than the total number of possible paths $l$. For one of our patients, the mean $l$ was 2852 , while $k$ was on average 61 . Currently, each bin comprises an equal distance interval, namely $0.5 \mathrm{~mm}$. The search is fastest when the size of the list per bin is balanced. Building the list of possible paths takes around 15 minutes.

\section{$6 \quad$ Results}

The user interface developed for visualization is shown in the top image of Figure 3. The desired safety margins to the ventricles and the blood vessels can be set on the right side. The remaining safe entry points and paths are then visualized together with the ventricles and blood vessels.

The bottom image in Figure 3 shows the user interface provided to inspect a selected path using a probe's eye view (MPR perpendicular to the path). Projections of the ventricle and blood vessel segmentations are shown on the reformatted data. The chosen safety margins are visualized as two circles and other distances can be measured by setting markers using the mouse. Meanwhile, the $3 \mathrm{D}$ context of the plane is shown to the right. The path's frame coordinates are presented in the bottom left corner.

In conclusion, the neurosurgeons now dispose of an automatic calculation of paths that avoid critical structures, of which the results are presented in 3D. Compared with the conventional path planning in $2 \mathrm{D}$, this gives a more orderly overview of safe entrance regions and therefore speeds up the planning procedure.

\section{Preliminary Validation}

A qualitative validation has been performed to check whether the planning is indeed fastened by the developed algorithm. The procedure consisted of a 


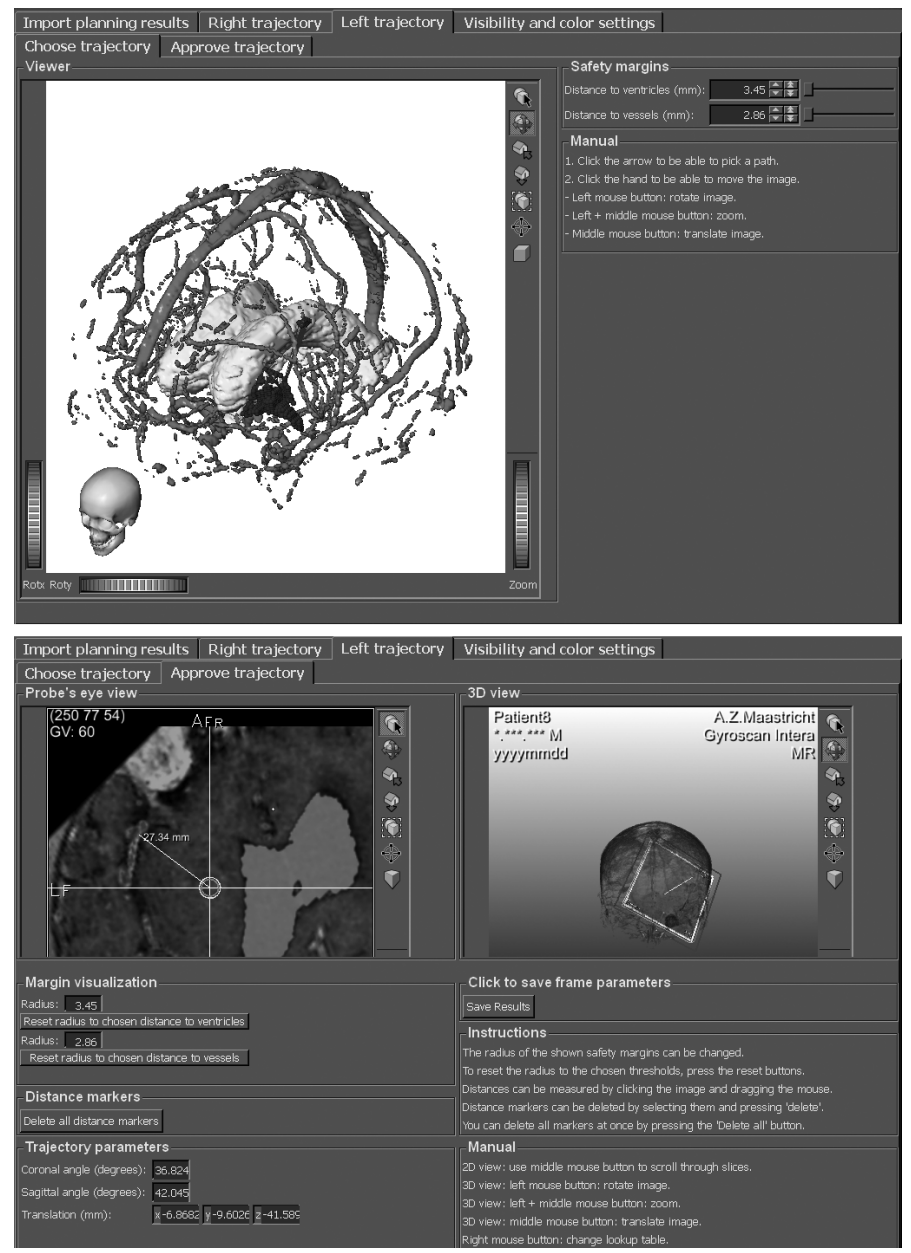

Fig. 3. User interfaces for visualization and inspection of paths. Top: paths together with ventricles and vessels. Bottom: inspection of selected path.

demonstration to one neurosurgeon and two resident neurosurgeons working in Maastricht University Hospital, with 10, 4, and 2 years experience with DBS, respectively. The neurosurgeons were not extensively trained on the new system. One resident attempted a planning himself after the demonstration. This took him about 25 minutes, while he needed on average about 45 minutes on the old system.

Subsequently, all three neurosurgeons were asked to fill out a questionnaire, containing questions about the functionality, appearance, and complexity of the system and its parts. A summary of their answers can be found in Table 1 .

Evidently, the neurosurgeons have received the application with considerable enthusiasm. The average score (over 17 questions and 3 neurosurgeons) is 3.9, 
Table 1. Average scores of questionnaire for qualitative validation. Scores were given on a scale of 1 to 5 , where 1 means 'very poor' and 5 represents 'excellent'. Surgeon 1 has 10 years of experience, while surgeon 2 and 3 have 4 and 2 years, respectively.

\begin{tabular}{|l|c|c|c|c|}
\hline & Surgeon 1 & Surgeon 2 & Surgeon 3 & Mean \\
\hline Path visualization (3 questions) & 3.33 & 5.00 & 4.33 & 4.22 \\
\hline Path approval (2 questions) & 4.50 & 4.00 & 3.50 & 4.00 \\
\hline General (4 questions) & 4.00 & 4.00 & 3.75 & 3.92 \\
\hline Improvement compared to current system & yes & yes & yes & - \\
\hline Amount of time to be gained (min) & 40 & 20 & 30 & 30 \\
\hline
\end{tabular}

and looking at the subapplication for path visualization and inspection, which was the main focus of this work, the mark increases to 4.1 .

The neurosurgeons all think that automatic calculation and $3 \mathrm{D}$ visualization is an improvement compared to the currently used planning systems. They expect time gain of 30 minutes. Considering timing statistics for 380 procedures of STN DBS in 12 different centers, collected by the participating neurosurgeons [13, revealing a mean planning time of 1 hour and 3 minutes with a standard deviation of 29 minutes, the necessary planning time could be halved.

\section{Discussion}

In this study, the feasibility of a faster path calculation for STN DBS has been investigated. We use a combination of existing algorithms to segment the ventricles, blood vessels, and gyri. Cost functions are calculated from these segmentations and used to automatically determine the possible paths to the planned target, together with their distances to ventricles and vessels. Afterwards, the segmentations and paths are visualized in 3D, where the neurosurgeon has the possibility to change safety margins interactively. A suitable path can be selected and examined in a probe's eye view. Subsequently, the required frame parameters are calculated.

The participating neurosurgeons are enthusiastic, estimating the time gain to be 30 minutes, about half of the current procedure. They have many suggestions to improve the appearance and functionality of the current application. For example, they would like to optimize the visualization, using more anatomical information of the head and the basal ganglia, and would like to develop the path inspection further. Furthermore, an extensive quantitative validation has to be performed, to assess the accuracy and utility of the proposed system regarding the registration and segmentation steps.

Until now, we have performed a manual registration, because the inevitable inaccuracy was not important for a feasibility study. However, automating this step using 3D rigid registration based on for example mutual information, will be implemented and validated in the near future.

Validation of the segmentation of critical structures is not trivial, because a gold standard for the ventricles and blood vessels is not readily available. 
Validation of the ventricles could be done using manual segmentations generated by collaborating physicians. For blood vessels a phantom could be used. However, the time-consuming generation of a ground truth and the subsequent validation were beyond the scope of this feasibility study.

For now, we projected the anatomical segmentations onto the probe's eye view visualization, as displayed in Figure 3 (bottom). This shows that the segmentation of the superior body of the ventricles is rather good. Nevertheless, looking at Figure 2 (left), it can be seen that the inferior horns of the ventricle are missing. The contrast between these horns and their surroundings is less and sometimes parts are invisible, probably due to partial volume effects. This causes gray value-based region-growing to miss these pieces. Therefore, it would be better to use model-based segmentation for this purpose. However, the inferior horns are not very important for STN DBS planning.

Regarding the blood vessels, the overlay in Figure 3 (bottom) and the segmentation in Figure 2 (right) are less convincing. The segmentation is noisy and the anterior part of superior sagittal sinus misses. However, we are aware of the fact that the used vesselness measure is not ideal, for example because it misses vessel junctions, and are planning to improve this in the near future.

In conclusion, every step in our prototype, from indirect targeting via rigid registration and vessel segmentation to the eventual visualization, can probably be replaced by a more sophisticated and better validated one. However, each of these steps constitutes in itself a field of research on which you can spend many years, while the presented work is just a feasibility study.

Apart from improvements on automatic avoidance of critical structures, another important subject for further research in this field is the STN targeting procedure. To save more time, the indirect atlas-based targeting should be replaced by an automatic segmentation of the target.

\section{References}

1. Benabid, A.: Deep brain stimulation for Parkinson's disease. Curr. Opin. Neurobiol. 13, 696-706 (2003)

2. Nowinski, W., Yang, G., Yeo, T.: Computer-aided stereotactic functional neurosurgery enhanced by the use of the multiple brain atlas database. IEEE Tr. Med. Im. 19(1), 62-69 (2000)

3. Lee, J., Huang, C., Lee, S.: Improving stereotactic surgery using 3-D reconstruction. IEEE Eng. Med. Biol. 21(6), 109-116 (2002)

4. Bourbakis, N.G., Awad, M.: A 3-D visualization method for image-guided brain surgery. IEEE Tr. Systems, Man, Cybernetics - Part B 33(5), 766-781 (2003)

5. Fujii, T., Emotoa, H., Sugoub, N., Mitob, T., Shibata, I.: Neuropath planner automatic path searching for neurosurgery. CARS: ICS 1256(587), 596 (2003)

6. Vaillant, M., Davatzikos, C., Taylor, R.H., Bryan, R.N.: A path-planning algorithm for image-guided neurosurgery. In: Troccaz, J., Mösges, R., Grimson, W.E.L. (eds.) CVRMed-MRCAS 1997, CVRMed 1997, and MRCAS 1997. LNCS, vol. 1205, pp. 467-476. Springer, Heidelberg (1997)

7. Weickert, J.: Coherence-enhancing diffusion filtering. Int. J. Comp. Vis. 31(2/3), 111-127 (1999) 
8. Géraud, T., Bloch, I., Maître, H.: 3D segmentation of brain structures from MR images using morphological approaches. Extract from 'Segmentation des structures internes du cerveau en imagerie par résonance magnétique'. $\mathrm{PhD}$ thesis, École Nationale Supérieure des Télécommunications, Paris, France (1998)

9. Schnack, H.G., Pol, H.E.H., Baaré, W.F.C., Viergever, M.A., Kahn, R.S.: Automatic segmentation of the ventricular system from MR images of the human brain. NeuroImage 14, 95-104 (2001)

10. Lohmann, G.: Extracting line representation of sulcal and gyral patterns in MR images of the human brain. IEEE Tr. Med. Im. 17(6), 1040-1048 (1998)

11. Frangi, A., Niessen, W., Vincken, K., Viergever, M.: Multiscale vessel enhancement filtering. In: Wells, W.M., Colchester, A.C.F., Delp, S.L. (eds.) MICCAI 1998. LNCS, vol. 1496, pp. 130-137. Springer, Heidelberg (1998)

12. Dijkstra, E.: A note on two problems in connexion with graphs. Numerische Mathematik 1, 269-271 (1959)

13. Coenen, V., Visser-Vandewalle, V.: Towards a standard of surgical care for Deep Brain Stimulation in Parkinson Disease: a 380 patients, multi center experience. Journal of Neurosurgery (submitted 2007) 